\title{
Transport and evolution of ion gyro-scale plasma blobs in perpendicular magnetic fields
}

\author{
P W Gingell ${ }^{1}$, S C Chapman ${ }^{1}$, R O Dendy ${ }^{2,1}$ and C S Brady ${ }^{1}$ \\ ${ }^{1}$ CFSA, Department of Physics, University of Warwick, Coventry, CV4 7AL, UK \\ ${ }^{2}$ Euratom/CCFE Fusion Association, Culham Science Centre, Abingdon, OX14 \\ $3 \mathrm{DB}, \mathrm{UK}$ \\ E-mail: p.gingell@warwick.ac.uk
}

\begin{abstract}
Strongly localised concentrations or depressions of plasma density and magnetic field strength ("blobs") are ubiquitous in the edge region of tokamak fusion experiments. The existing fusion plasma literature in this area focuses primarily on blobs sufficiently large that a fluid description is appropriate. However, the blob population may include some - not necessarily easily detectable - whose characteristic lengthscales are on the order of the ion gyro-scales. This implies that a description at the fluid level is unlikely to capture the full dynamics. Here, therefore, we report hybrid (particle ions, fluid electrons) simulations of ion gyro-scale blobs, which enable us to examine the effects of finite Larmor radius on their dynamics and evolution. We find that ion gyro-scale blobs are advected with the background flow, and develop a twin-celled vortex structure. Asymmetry then arises from finite ion Larmor radius kinetics, manifesting in the size of the internal vortices, the shape of tails forming from blob ejecta, and the growth of a Kelvin-Helmholtz instability.
\end{abstract}

PACS numbers: $52.65 . \mathrm{Ww}, 52.55 . \mathrm{Fa}$

Submitted to: Plasma Phys. Control. Fusion

\section{Introduction}

The outer regions of tokamak plasmas, near the last closed flux surface, are observed to generate coherent propagating blobs. These undergo radial transport, and can break free of the confinement region. Blobs with sufficient radial velocity can strike 
the walls of the tokamak, increasing local particle and energy fluxes, reducing divertor efficiency, and increasing impurity levels. Such blobs have been imaged in, for example the DIII-D [1], Alcator C-mod [2], NSTX [3], ASDEX Upgrade [4] and MAST [5] tokamaks and in other laboratory experiments, for example [6]. Some blobs may be the poloidal projection of magnetic flux tubes, or ropes, that extend along the direction of the magnetic field. Polarising curvature drifts of ions and electrons, giving rise to an electric field that may not be shorted out by parallel motion under tokamak edge conditions, give rise in the classic picture [7] to $\mathbf{E} \times \mathbf{B}$ radial blob motion. Models for blob transport and evolution have principally focused on fluid and multi-fluid descriptions, approached both analytically and numerically; see, for example, [8-13]. These studies demonstrate a range of evolutionary phenomena, such as mushrooming, fingering due to interchange instabilities, Kelvin-Helmholtz vortex shedding, and variable scales for coherence with dependence on dissipation levels. There have been successful simulations of blob creation via interchange-ballooning modes [9]. For a recent review of edge plasma physics in tokamaks we refer to [14].

Current tokamak diagnostics offer clear observations of large scale blobs, however their limited resolution at present prohibits the observation of smaller blobs on scales of the order of the ion gyro-radius. In such cases, ion particle dynamics may play an important role in blob transport and evolution, inviting a kinetic numerical study. For plasma phenomena arising on the smallest length and time scales, kinetic methods such as particle-in-cell (PIC) codes are applicable. However the ion gyro-scales of interest here represent an intermediate phase between fully kinetic treatments, and previous studies in the fluid regime. On these intermediate scales, it is necessary to capture the gyration of ions around magnetic field lines, while the gyration of electrons can be neglected. Hence a hybrid model is required for which ions are treated kinetically, and electrons as a fluid.

A hybrid model has numerous advantages which apply directly to the results presented here. These include: capturing the non-linear interaction between ion gyration and plasma inhomogeneity; cross-scale coupling between ion gyro-scale kinetic modes and fluid MHD-like modes; and the ability to resolve phenomena on much shorter timescales than fluid models. In addition to the ability to study inhomogeneities in configuration space, hybrid codes also enable the study of a non-Gaussian ion velocity distributions, such as beams and their instabilities, while incorporating several distinct ion populations. Interaction and momentum transfer between these phase space populations can lead to novel results that distinguish a 
hybrid model from a purely fluid one.

The first true hybrid code, comprising particle ions and fluid electrons, was applied to pinch experiments in laboratory plasmas [15]. Among the first problems addressed subsequently using hybrid codes was the identification of heating mechanisms in collisionless shocks, such as the Earth's bow shock [16]. Hybrid codes have since been applied to the study of quasi-perpendicular shocks, reproducing phenomena such as ion reflection and overshoots of magnetic field and density [17]. Applications include the interaction of the solar wind with both strongly magnetic obstacles and weakly magnetic obstacles. These simulations reveal a potential issue with hybrid codes, in the anomalous shock reformation caused by the gyration of ions upstream $[18,19]$. Targets of hybrid code study include strongly interacting bodies such as the Earth's magnetosphere [20], and weakly interacting bodies such as Mars [21], Venus [22], comets [23] and Titan [24]. Artificial solar wind obstacles, such as the AMPTE ion releases [25], have also received considerable attention [26]. Hybrid codes have also been used to simulate collisionless magnetic reconnection [27-29], allowing the study of kinetic effects on the ion tearing and beam instabilities, and asymmetries which develop in the magnetic structure of thin current sheets [30]. Although hybrid codes incorporating six-dimensional particle-in-cell ion kinetics are thus widely applied to the study of space plasmas, this paper represents perhaps their first application to distinctive magnetic confinement fusion plasma regimes. In the fusion context, hybrid treatments [31] typically involve ion kinetics that are gyro-averaged.

Simulations within the field of space plasma physics which most closely resemble the work presented here are those which deal with small unmagnetised objects in the solar wind $[23,25,26]$. These simulations have predominantly addressed obstacles in fast, super-Alfvénic flows, with an obstacle size $R \ll \rho_{i}$, the ion gyro-radius. Here we present simulations for an unexplored intermediate range, applicable to tokamak blobs, for which we have a slow, sub-Alfvénic background flow, and obstacle size $R \gtrsim \rho_{i}$.

In this paper we examine the transport and evolution of ion gyro-scale blobs within the confined plasma, ranging from $\rho_{i}$ to $10 \rho_{i}$ in radius, where the background ion gyro-radius $\rho_{i}=\sqrt{2 m_{i} k T_{i}} / q B_{0}$ for ions of mass $m_{i}$ in a population with temperature $T_{i}$. We discuss the phenomena which arise both as a result of fluid-like bulk flow and the interaction of ions with gyro-scale inhomogeneities. These features include the advection of the blob with the flow, the development of an internal 
twin-celled vortex pattern, and Kelvin-Helmholtz instability in the tail produced downstream of the blob. The blobs are found to evolve asymmetrically due to the effects of finite ion Larmor radius, with a stronger effect for blobs of smaller radius.

\section{Hybrid Model}

A detailed description of the general hybrid method can be found in [32]. The hybrid model treats ions kinetically as particles, with continuous positions in phase space, acted upon by the Lorentz force. Electric and magnetic fields are evolved self-consistently to second order accuracy in time and space, on a staggered grid. To derive this model, the following assumptions are made: inertia-less electrons; $\nabla \cdot \mathbf{E}$ is negligible on length scales of interest, implying charge neutrality such that $e n_{e}=q_{i} n_{i}$ for a single ion species $i$; collisionless plasma; and an ideal, isothermal electron gas, $\nabla \cdot \underline{\underline{P_{e}}}=\nabla p_{e}=k T_{e} \nabla n_{e}$. We take the Darwin limit [33] of Maxwell's equations, which neglects displacement current and hence eliminates fast electromagnetic modes.

The hybrid code updates the magnetic field using Faraday's law, and the electric field using the electron fluid momentum equation:

$$
n_{e} m_{e} \frac{\mathrm{d} \mathbf{v}_{e}}{\mathrm{~d} t}=-e n_{e}\left(\mathbf{E}+\mathbf{v}_{e} \times \mathbf{B}\right)-\nabla \cdot \underline{\underline{P_{e}}} .
$$

Here $m_{e}$ is the electron mass, $n_{e}$ is the electron fluid number density, $\mathbf{v}_{e}$ is the electron fluid velocity, $\underline{\underline{P_{e}}}$ is the electron pressure tensor, and $\mathbf{E}$ and $\mathbf{B}$ are the electric and magnetic fields respectively. This equation neglects any resistive coupling between the electrons and the ion particle species. We now treat the electron fluid as inertialess, such that the left hand side of (1) is zero. Eliminating the electron fluid velocity using Ampère's law in the Darwin limit,

$$
\nabla \times \mathbf{B}=\mu_{0} \mathbf{J}=\mu_{0} q_{i} n_{i}\left(\mathbf{v}_{i}-\mathbf{v}_{e}\right),
$$

(1) gives the following Ohm's Law, which is used to update the electric field:

$$
\mathbf{E}=-\frac{1}{e n}\left(\frac{q_{i}}{e} k T_{e} \nabla n+\frac{\nabla B^{2}}{2 \mu_{0}}-\frac{(\mathbf{B} \cdot \nabla) \mathbf{B}}{\mu_{0}}+\mathbf{J}_{i} \times \mathbf{B}\right),
$$

where $n$ is the number density of both the ions and the electron fluid, $T_{e}$ is the electron fluid temperature, and $\mathbf{J}_{i}$ is the ion current density. It is important to note that, under these assumptions, electric fields cannot arise from charge separation; hence 
electric fields are purely motional in origin. This level of model description allows for the propagation of Alfvén and magnetoacoustic waves, ion cyclotron waves, lower hybrid waves and whistler waves. However, since whistler waves are undamped at the electron gyrofrequency due to the neglect of electron gyration, numerical damping must be applied to the electromagnetic fields to prevent their unstable growth.

\section{Initial Conditions and Simulation Geometry}

For the hybrid model presented here, each ion follows a trajectory in a six dimensional phase space. Vector fields $\mathbf{E}, \mathbf{B}$ and $\mathbf{J}$ vary in two dimensional configuration space $(x, y)$ and in time, and are evolved on a staggered two dimensional Cartesian grid with three components at each grid point, e.g. $E_{x, y, z}(x, y, t)$.

The grid size is chosen to be smaller than all ion gyro-scales in the system by approximately an order of magnitude. The box size is then chosen to be sufficiently large to capture the full evolution of the moving plasma blob and is typically 450 ion Larmor radii. The time step is determined by CFL condition and is on the order of $10^{5}$ time steps per ion gyro-period. We use sufficient particles per cell to fully represent the ion velocity distribution and a typical simulation is $2 \times 10^{7}$ total computational particles.

Blobs are set up as flux ropes with Gaussian spatial profiles for enhancements of particle number density, and depressions of magnetic field strength out of the simulation plane, $B_{z}$ (Figure 1). The magnitudes of the Gaussian enhancements and depressions are chosen such that blobs are set up in pressure equilibrium with the background flowing plasma, balancing combined gas and magnetic pressures. The incorporation of a finite Larmor radius kinetic description for the ions, however, leads to a small force imbalance for particles in inhomogeneous regions. The background plasma parameters are chosen to be approximately characteristic of edge conditions in a medium-size tokamak such as MAST, with number density $n_{0}=10^{19} \mathrm{~m}^{-3}$, magnetic field $B_{z, 0}=0.4 \mathrm{~T}$, ion and electron temperature $T_{0}=4 \times 10^{6} \mathrm{~K}$, and ion

gyro-radius $\rho_{i}=8 \times 10^{-3} \mathrm{~m}$. Other parameters, notably those characterising the blobs and the background flow speed, are chosen with a view to achieving significant temporal evolution given the spatial resolution required and the computational resource available. The background is initialised flowing transversely to the magnetic field at speed $u_{0}=0.2 v_{A}$, and the simulations are carried out in the initial rest frame of the blob. Conversely in the rest frame of the background flow, the blob initially 


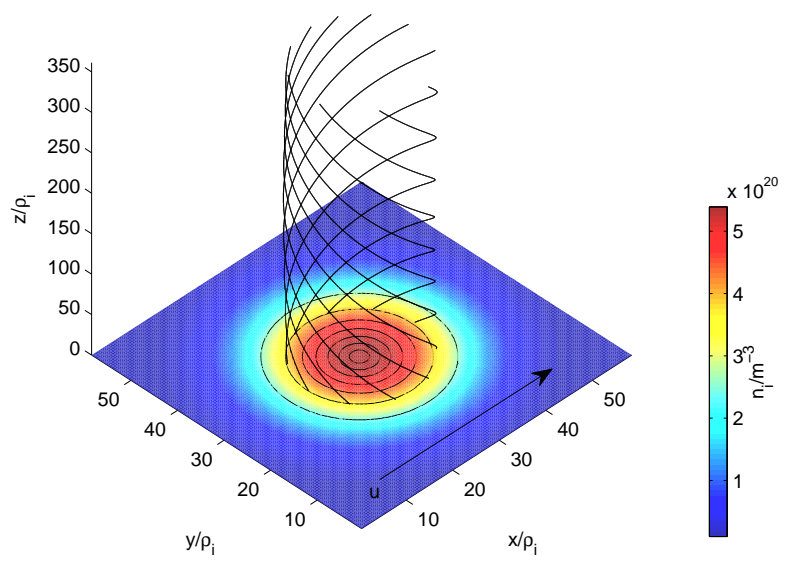

Figure 1. Initial conditions of the simulated blobs. Magnetic field lines are plotted in black, extended out of the simulation plane to display the flux rope structure, with the number density in colour for a blob of radius $10 \rho_{i}$.

moves transversely to the magnetic field with velocity $-u_{0}$. Numerical and physical parameters are listed comprehensively in Table 1.

With the initial $B_{x, y}$ magnetic field components of the flux rope structure being much smaller than the perpendicular $B_{z}$, wave modes are dominated by those which propagate for $\mathbf{B} \perp \mathbf{k}$. Thus magnetoacoustic waves dominate over Alfvén and whistler waves. Likewise, the magnetic tension force $(\mathbf{B} \cdot \nabla) \mathbf{B}$ is small compared to the magnetic pressure force $\nabla\left(B^{2} / 2\right)$

The evolution of six different blobs is analysed in this paper, of varying radius from $\rho_{i}$ to $10 \rho_{i}$. In each case, the central blob values of magnetic field strength and density are the same, such that the total mass of the blobs scales with the square of their radii.

\section{Simulation Results}

Here we present the results of hybrid code simulations of the evolution of ion gyroscale blobs over several ion gyroperiods. The simulations reveal five key elements 
Table 1. Physical and numerical parameters for blob simulations in this paper.

\begin{tabular}{ll}
\hline Parameter & \\
\hline Background ion gyro-radius, $\rho_{i}$ & $8 \times 10^{-3} \mathrm{~m}$ \\
Background ion gyro-period, $t_{\Omega}$ & $1.6 \times 10^{-7} \mathrm{~s}$ \\
Spatial resolution, $\Delta x, \Delta y$ & $0.4 \rho_{i}$ \\
Simulation domain & $960 \Delta x, 450 \rho_{i}$ \\
Total particles & $2 \times 10^{7}$ \\
Background magnetic field, $\mathbf{B}_{0}$ & $0.4 \mathrm{~T}$ \\
Internal magnetic field, $\mathbf{B}_{\mathrm{blob}}$ & $0.08 \mathrm{~T}$ \\
Background number density, $n_{0}$ & $10^{19} \mathrm{~m}^{-3}$ \\
Peak number density, $n_{\mathrm{blob}}$ & $55 n_{0}$ \\
Temperature, $T_{0}$ & $4 \times 10^{6} \mathrm{~K}$ \\
Background ion gyro-period, $t_{\Omega}$ & $1.6 \times 10^{-7} \mathrm{~s}$ \\
Alfvén speed, $v_{A}$ & $2.8 \times 10^{6} \mathrm{~ms}^{-1}$ \\
Sound speed, $c_{s}$ & $2.3 \times 10^{5} \mathrm{~ms}^{-1}$ \\
Internal plasma beta, $\beta_{\text {blob }}$ & 12 \\
Background plasma beta, $\beta_{\text {flow }}$ & 0.01 \\
Electron Debye length, $\lambda_{D}$ & $4.4 \times 10^{-5} \mathrm{~m}$ \\
\hline
\end{tabular}

of the evolution, to be discussed at greater length below: the advection of the blobs downstream in the background flow; the development of an internal twincelled convection pattern; the growth of ion gyro-scale structures, both internal and external to the evolving blob; asymmetry in the growth of a tail behind the blob; and the formation of an asymmetric Kelvin-Helmholtz instability in the tail.

The analysis begins with a description of the bulk evolution for a blob with radius $R_{b} \gg \rho_{i}$, for which fluid approximations are more appropriate, including the advection due to momentum transfer from flow to blob and the development of the twin convection vortices inside the blob. A detailed account of the asymmetry which develops in these vortices for smaller blobs with radius $R_{b} \sim \rho_{i}$ follows, together with a discussion of features unique to those blobs. Finally we examine the formation of the asymmetric Kelvin-Helmholtz instability and its dependence on blob radius.

\subsection{Fluid-like Phenomena}

Snapshots of the blobs in ion number density as they evolve in time are given for $R_{b} \gg \rho_{i}$ blobs in Figure 2. The clearest features of the evolution of these blobs, with 
radii ranging from 6 to 10 ion gyro-radii, are related to bulk plasma flow rather than ion kinetic effects due to individual ion particle interactions. Two of these features, the advection of the blob and vortex generation, are described below.

\subsubsection{Bulk Evolution}

As a result of the difference in velocity between the blob and background plasma flow, the background plasma is seen to pile up on the upstream edge, resulting in an increase in the number density in that region. The interaction between this background plasma and the blob, here acting as an obstacle, leads to momentum transfer in the flow direction from the background to the blob. In the initial frame of reference of the blob, as shown in Figure 2, this leads to advection of the blob plasma in the flow direction. Trajectories of the centre of mass for blobs of radius $R_{b} \gg \rho_{i}$ are given in Figure 3. These trajectories display approximately symmetric advection of the large blobs, with a maximum deviation of approximately $0.1 \rho_{i}$ after seven ion gyro-periods.

In the case of an initially stationary blob of plasma with radius $R_{b} \gg \rho_{i}$ in a magnetic field, the expected solution under purely fluid assumptions is the deflection of background flow around the blob, with two oppositely directed vorticity cells within its upper and lower halves. Such a solution would be perfectly antisymmetric in the up-down direction (as in [34]). The vorticity cells may be generated in part by momentum transfer around perturbations which grow with the onset of KelvinHelmholtz instability at the shear boundary between stationary blob plasma and flowing background plasma. Calculated streamlines for the largest blob, of radius $10 \rho_{i}$, are given in Figure 4, and display the twin-celled vortex pattern.

\subsection{Kinetic Phenomena}

For blobs with radius $R_{b} \sim \rho_{i}$, snapshots of their ion number density as they evolve in time are given in Figure 5. The clearest features of the evolution of these blobs, whose radii range from 1 to 4 ion gyro-radii, are the growth of structures on the ion gyro-scale, and the introduction of asymmetries into the fluid-like phenomena. This asymmetry is most clear in the tails that form downsteam of the blob, with a larger, more dense tail forming on the lower edge of the blobs.

The asymmetry is also apparent in the trajectories of individual particles, as seen particularly for a blob of radius $2 \rho_{i}$ in Figure 6, but also for a blob of radius 

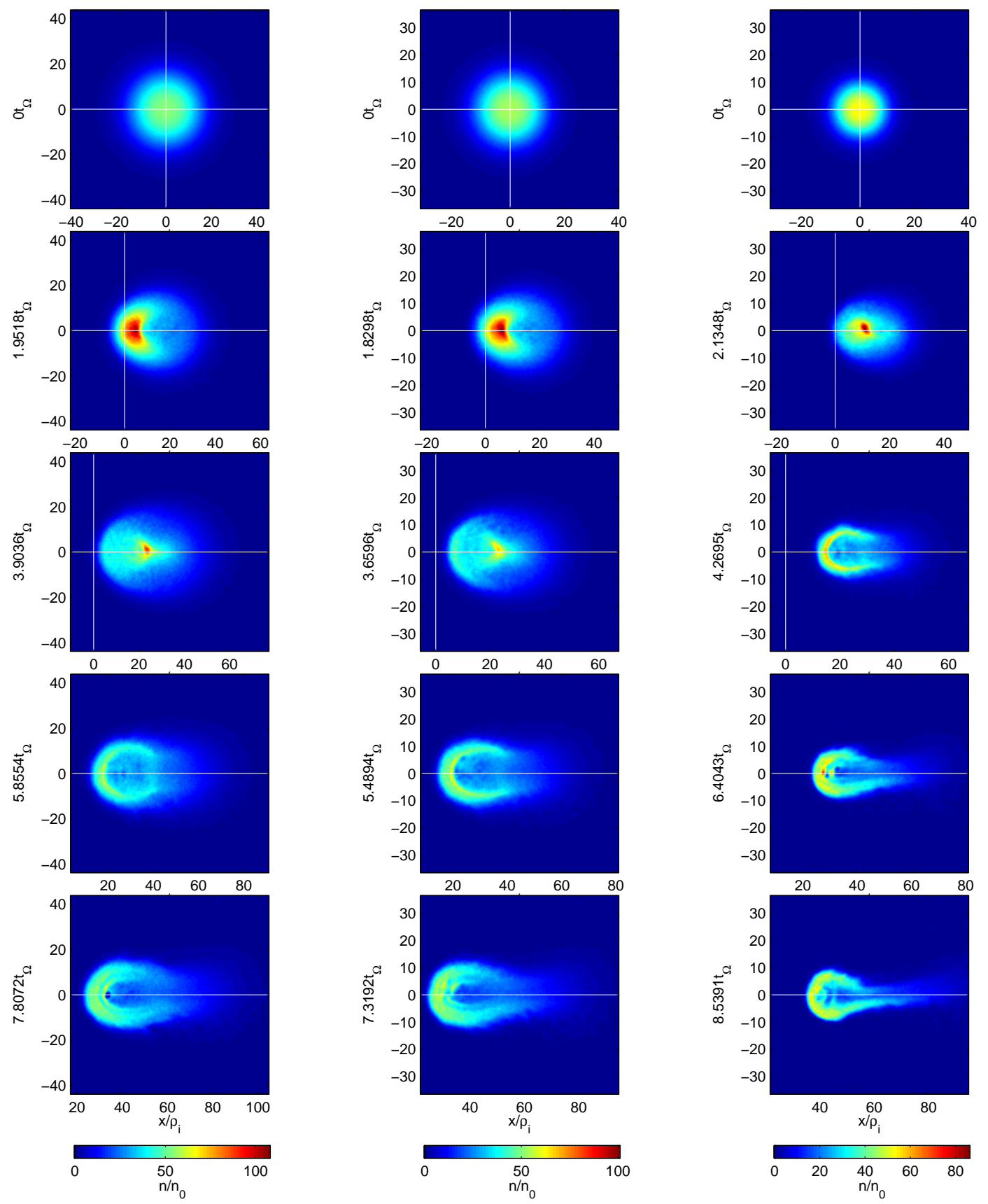

Figure 2. Number density colour maps for the time evolution of blobs with radii $R_{b} / \rho_{i}=10$ (left column), 8 (centre), 6 (right). The white lines mark the starting position of the blob in the $\mathrm{x}$ - and $\mathrm{y}$-direction. These larger blobs are advected to the right, develop an asymmetric tail, and evolve internal structures on the order of the ion gyroradius. At late times, a Kelvin-Helmholtz instability is seen to grow on the lower edges of the blobs. 


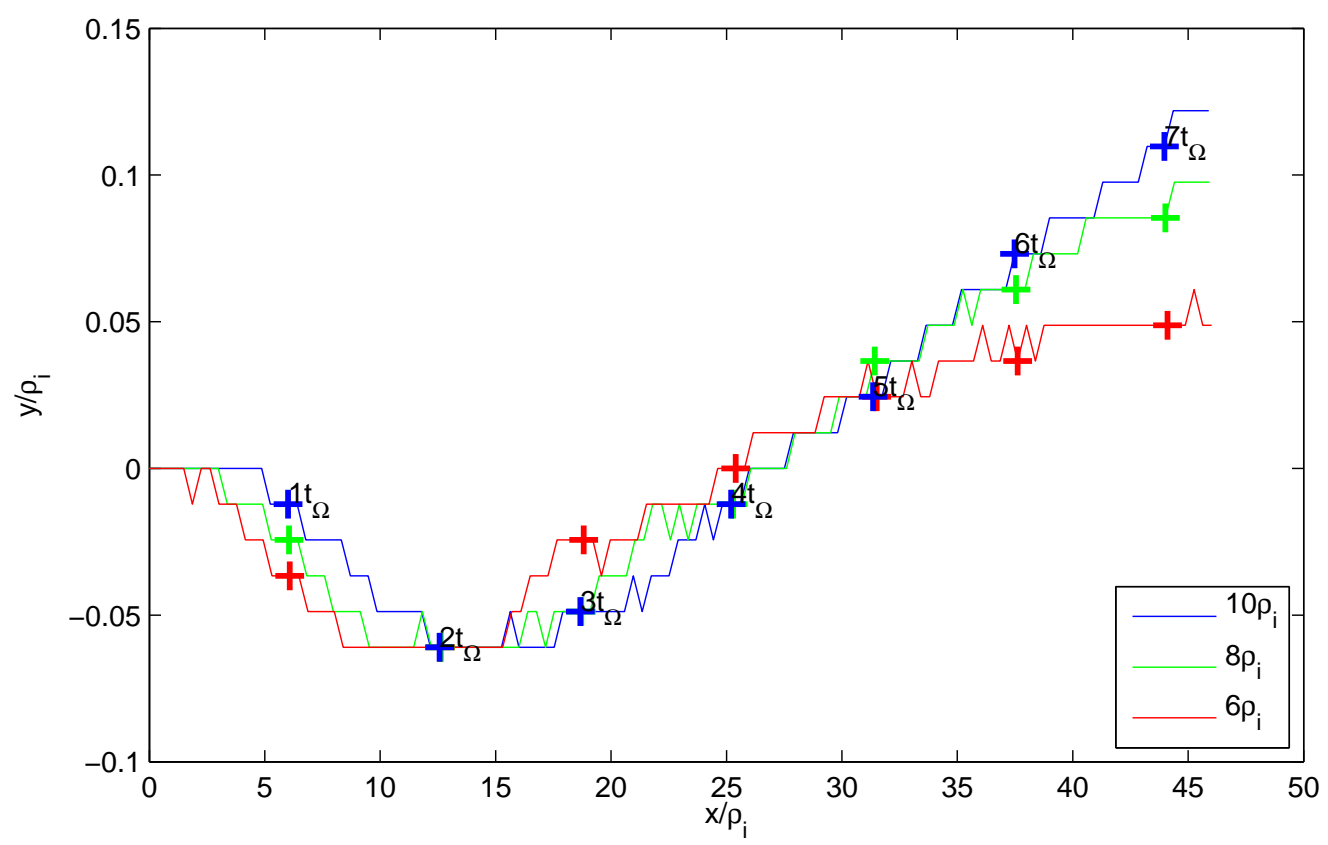

Figure 3. Trajectories in the $x-y$ plane of the centre of mass for blobs of radius 6-10 $\rho_{i}$ from $t=0$ until approximately 7 gyroperiods have elapsed. The centre of mass is calculated by taking moments over only those grid cells which have a number density greater than twice that of the background.

$10 \rho_{i}$. The underlying physics behind this asymmetry is a direct consequence of the inclusion of ion gyration in the hybrid model, and is described in the following section.

\subsubsection{Finite Larmor Radius Symmetry Breaking}

In the rest frame of the background flow, we have two distinct populations of ions in velocity space: these comprise the stationary background and the moving ion gyro-scale blob. Since an ion traverses much of the blob as it gyrates in the magnetic field, we expect gyromotion to play a significant role in the dynamics and evolution of both ion populations. The chirality of ion gyration can thus break the symmetry of the evolution of an otherwise symmetric blob. We can describe this asymmetry mathematically as follows. From the Vlasov equation,

$$
\frac{\partial f_{\alpha}}{\partial t}+\mathbf{v} \cdot \nabla f_{\alpha}+\frac{q_{\alpha}}{m_{\alpha}}\left(\mathbf{E}+\mathbf{v}_{\alpha} \times \mathbf{B}\right) \cdot \nabla_{\mathbf{v}} f_{\alpha}=0
$$




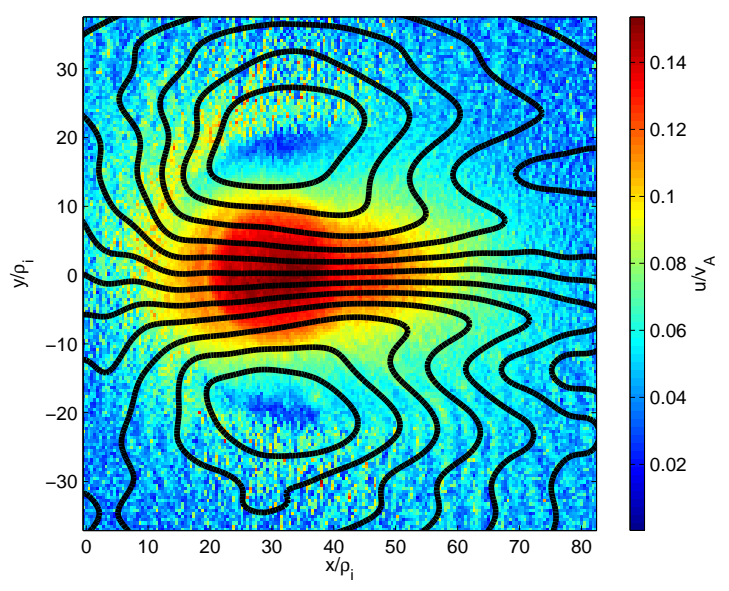

Figure 4. Approximate streamlines for a blob of radius $10 \rho_{i}$ calculated under the assumption of incompressible flow, $\nabla \cdot \mathbf{u}=0$. Streamlines are plotted in the rest frame of the flow, with the magnitude of the velocity also given in colour. Though we do not in fact have incompressible flow, these streamlines suffice to illustrate the two convection cells formed on the upper and lower sides of the blob by momentum transfer which may be due to Kelvin-Helmholtz instability.

taking the second moment by multiplying by $\mathbf{v v}$ and integrating over velocity space, we arrive at the bulk momentum equation for an ion species $\alpha$ :

$$
n_{\alpha} m_{\alpha} \frac{\mathrm{d} \mathbf{v}_{\alpha}}{\mathrm{d} t}=q_{\alpha} n_{\alpha}\left(\mathbf{E}+\mathbf{v}_{\alpha} \times \mathbf{B}\right)-\nabla p_{\alpha}
$$

Using the Ohm's law for our hybrid method (3), together with Ampère's law in the low frequency limit,

$$
\nabla \times \mathbf{B}=\mu_{0} \mathbf{J}=\mu_{0} e\left(\sum_{\alpha} n_{\alpha} \mathbf{v}_{\alpha}-n \mathbf{v}_{e}\right),
$$

and with a proton plasma, we can eliminate the electric field $\mathbf{E}$ and electron velocity $\mathbf{v}_{e}$ to express (5) in the form

$$
\begin{aligned}
m_{\alpha} n_{\alpha} \frac{D \mathbf{v}_{\alpha}}{D t}= & e n_{\alpha}\left(\mathbf{v}_{\alpha}-\frac{1}{n} \sum_{\alpha^{\prime}} n_{\alpha^{\prime}} \mathbf{v}_{\alpha^{\prime}}\right) \times \mathbf{B} \\
& +\frac{n_{\alpha}}{n}\left(\mathbf{J} \times \mathbf{B}-\nabla p_{e}\right)-\nabla p_{\alpha} .
\end{aligned}
$$



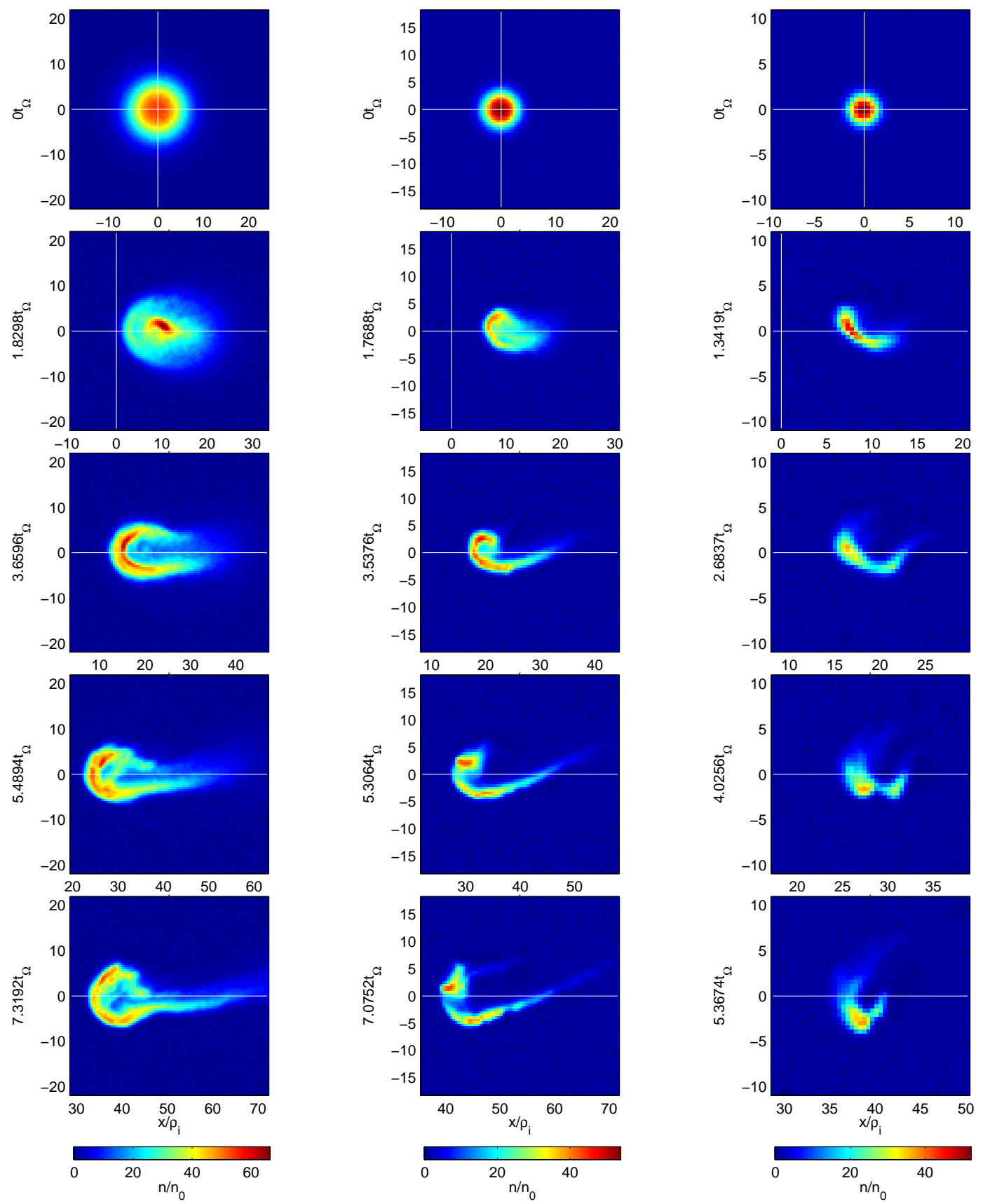

Figure 5. Number density colour maps for the evolution of a blob with radii $R_{b} / \rho_{i}=4$ (left column), 2 (centre), 1 (right). The white lines mark the starting position of the blob in the $\mathrm{x}$ - and $\mathrm{y}$-direction. The small blobs are advected to the right, along the direction of the background flow, and develop structures directly related to the gyration of individual ions. There is a clear up-down asymmetry in the growth of the tail that forms downstream of the blob as it evolves. 

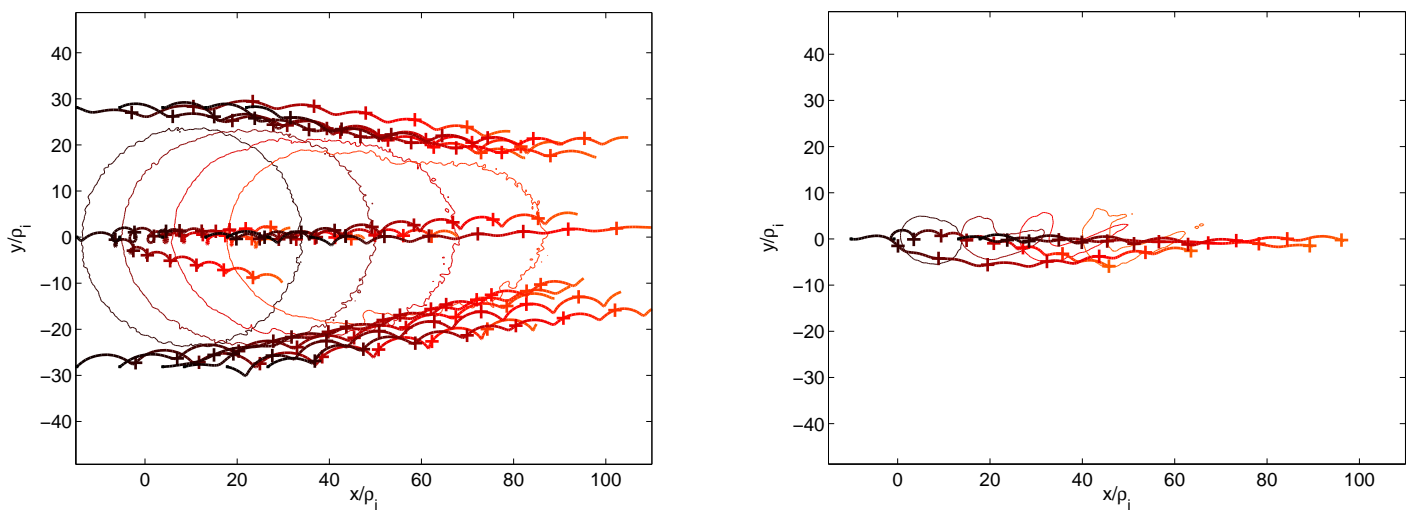

Figure 6. Particle trajectories in the $10 \rho_{i}$ blob (left) and $2 \rho_{i}$ blob (right), for particles positioned initially on lines on the centre, top and bottom, upstream and inside the blob, overlaid on contours of number density after 1, 3, 5 and 7 gyroperiods. The colour of the particle tracks and contours is related to the time, with black being the start of the simulation, and red the end. Ticks are given on the particle trajectories at each complete gyroperiod. Of those particles which begin on the central, symmetric line, many are pushed towards the lower edge of the blob by the FLR symmetry breaking effect within a few ion gyroperiods.

While the last terms on the RHS arise from conventional single fluid MHD forces for ion species $\alpha$, the first term on the RHS represents an asymmetry present only in spatially inhomogeneous regions in which there is a difference between the mean velocities of different populations of ions. In our case, these populations are the ions in the blob and those in the background plasma.

In order to understand the effect of the asymmetric term in (7) on a plasma with finite ion Larmor radius, we must first consider the effect of a moving frame of reference on individual particle dynamics, and the associated appearance of motional electric fields. Let us consider the two frames of reference shown in Figure 7. In Frame 1 the background plasma is stationary, with the blob plasma moving with velocity $\mathbf{v}$ in the $-x$ direction. Frame 2 is moving with velocity $\mathbf{v}$ with respect to Frame 1 , such that the background plasma is flowing with velocity $\mathbf{u}=\mathbf{- v}$ and the blob plasma is stationary. The simulations presented in this paper are carried out in Frame 2.

A test particle in the background plasma in Frame 1, with components of velocity in the $\mathrm{x}-\mathrm{y}$ plane, will gyrate with a circular trajectory around the perpendicular magnetic field lines as shown in Figure 8. However if we transform into Frame 2, 


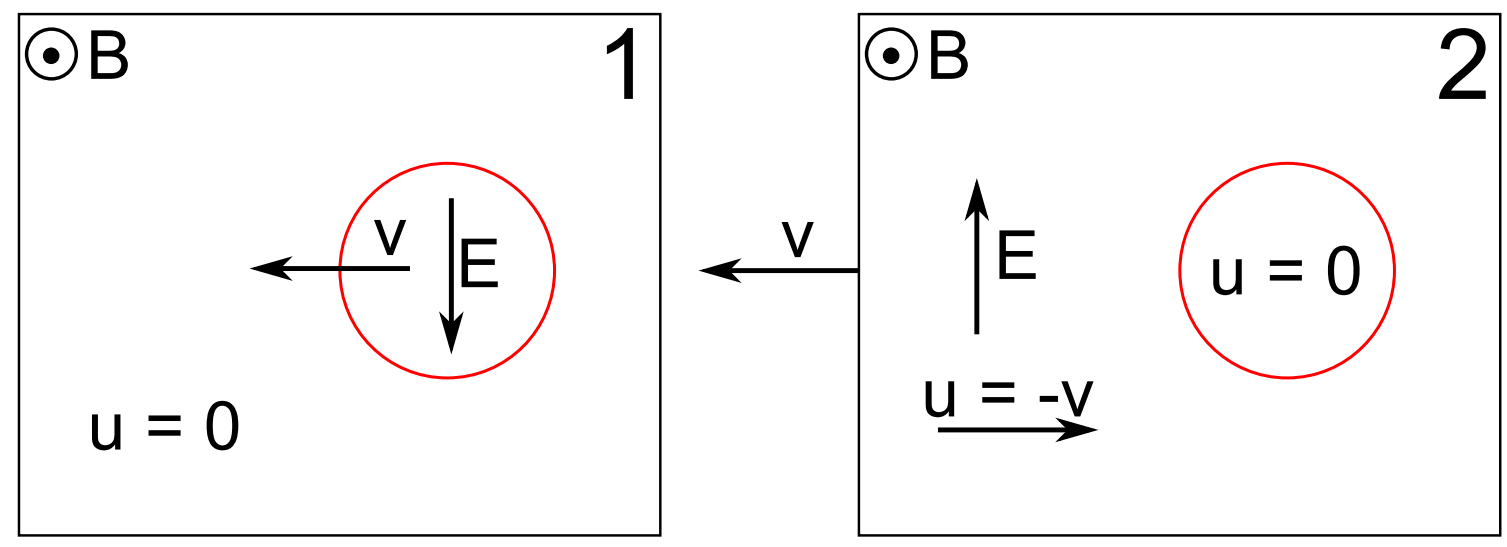

Figure 7. Vector directions internal and external to the blob (red) for the rest frames of the background flow plasma (Frame 1) and blob plasma (Frame 2).

the electromagnetic fields are subject to the following transformations in the nonrelativistic case:

$$
\begin{aligned}
& \mathbf{B}_{2}=\mathbf{B}_{1} \\
& \mathbf{E}_{2}=\mathbf{E}_{1}-\mathbf{v} \times \mathbf{B}_{1} .
\end{aligned}
$$

In Frame 2, charged particles in the background are thus subject to a motional electric field $\mathbf{E}_{2}$ which arises as a result of a frame moving relative to a magnetic field. If the frame velocity $\mathbf{v}$ is perpendicular to $\mathbf{B}$, the gyrating particle in Frame 1 is, in this moving frame, subject to an $\mathbf{E} \times \mathbf{B}$ drift, and demonstrates a cycloid orbit as shown in Figure 8. This describes the trajectories of background particles in the simulations, and the source of the background electric field $\mathbf{E}_{y}$ in the absence of charge separation.

Particles which are stationary in Frame 2, i.e. those initialised in the blob, are accelerated by this motional electric field if they leave the spatial region that is dominated by blob ions and enter the background flowing plasma. This acceleration occurs initially in the direction of the motional electric field $\mathbf{E}_{2}$, and leads to a momentum gain of the blob particles in the $\mathbf{v} \times \mathbf{B}=-\mathbf{u} \times \mathbf{B}$ direction. Ultimately, such particles are "picked-up" by the background flow, with a cycloid trajectory in the $\mathbf{E}_{\mathbf{2}} \times \mathbf{B}$ direction with gyro-radius $2 u_{0} / \Omega_{\mathrm{c}, \mathrm{i}}$. 

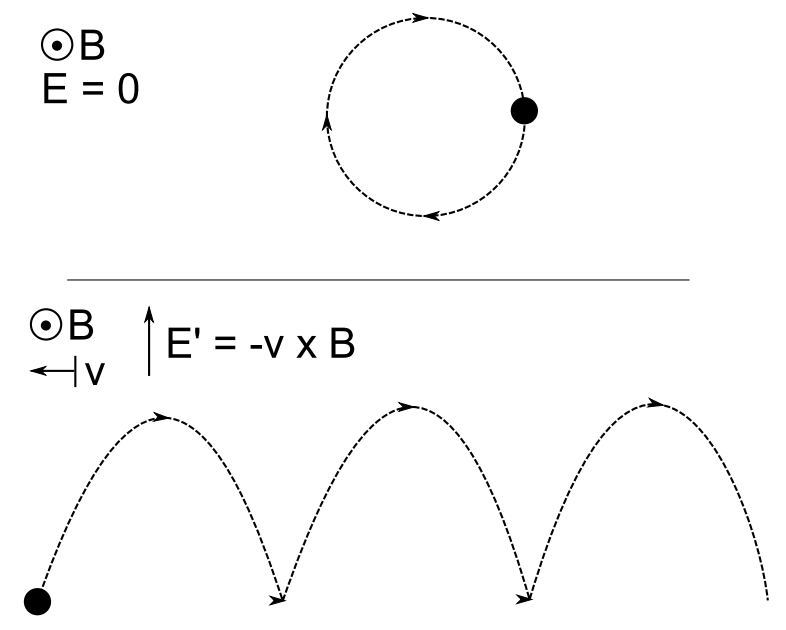

Figure 8. Single particle trajectories for an ion with velocity perpendicular to a background magnetic field in a stationary (top) and moving (bottom) frame of reference.

Following the same analysis in the rest frame of the background flow, Frame 1 , such that the blob is moving with velocity $\mathbf{v}$ and particles in the background are initially stationary, it becomes clear that in this frame, a motional electric field $\mathbf{E}=-\mathbf{v} \times \mathbf{B}=\mathbf{u} \times \mathbf{B}$ exists within the blob. Hence, particles initially within the stationary flow, which subsequently enter the spatial region dominated by moving blob ions, are there accelerated in the $\mathbf{E}_{1}=-\mathbf{E}_{2}$ direction and proceed with cycloid orbits in the $\mathbf{E}_{1} \times \mathbf{B}$ direction. We emphasise again that the hybrid code calculate particle-field interactions directly and self-consistently at the level of each individual ion.

The overall effect of these motional electric fields is a deflection of the blob plasma in the $-\mathbf{u} \times \mathbf{B}(+y)$ direction at the upstream edge of the blob, and a deflection of the background flow plasma in the $\mathbf{u} \times \mathbf{B}(-y)$ direction. A net momentum gain for the blob or flow ion population in the $y$ direction can only occur for finite Larmor radius ions: the gyration caused by the perpendicular magnetic field breaks the symmetry that would result from the asymmetric term in (7) under multifluid assumptions including zero Larmor radius. This form of finite Larmor radius symmetry breaking has been observed in the AMPTE artificial comets released into the solar wind in 1984 [25]. 


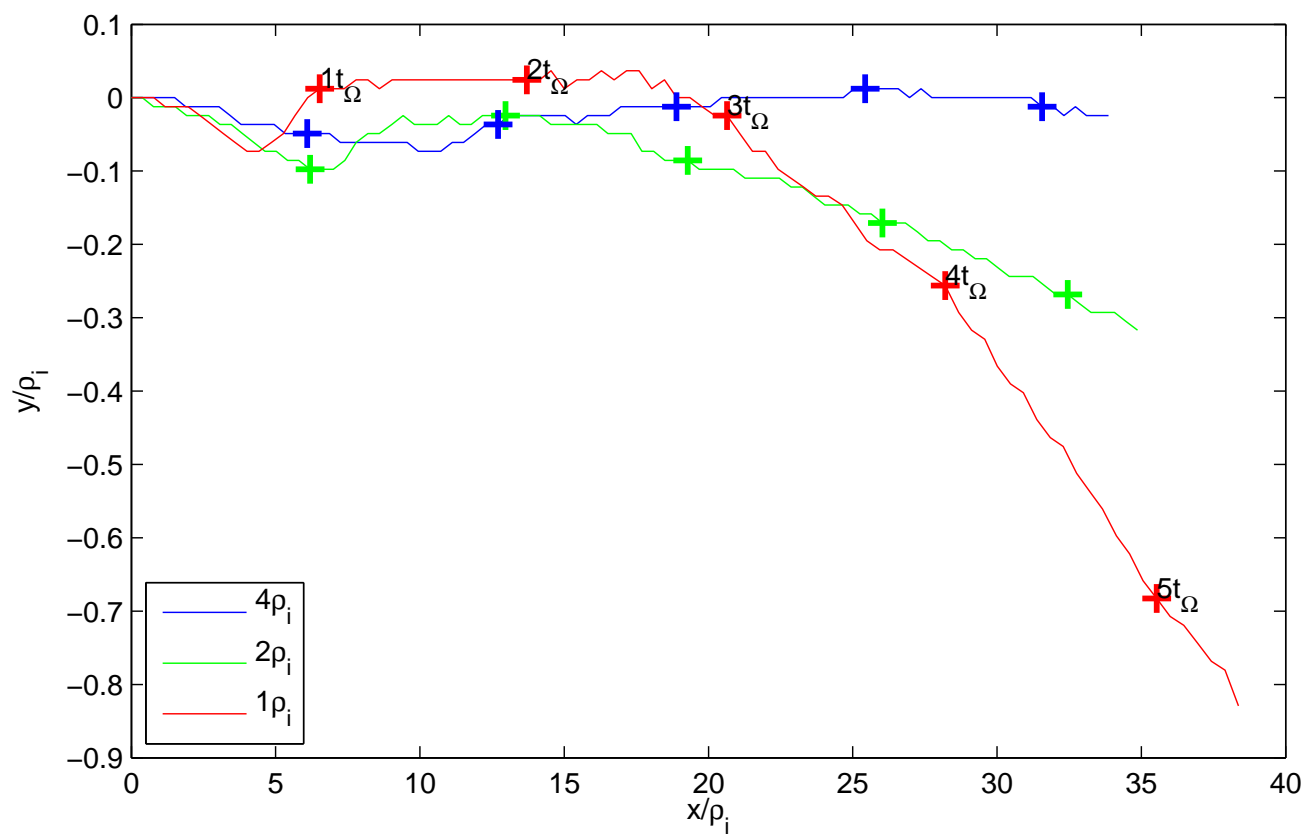

Figure 9. Trajectories in the $x-y$ plane of the centre of mass for blobs of radius $1-4 \rho_{i}$ from $t=0$ until approximately 5.5 gyroperiods have elapsed. The centre of mass is calculated by taking moments over only those grid cells which have a number density greater than twice that of the background. There is greater poloidal deflection for smaller blobs.

\subsubsection{Asymmetry in Bulk Evolution}

As noted previously, blobs are advected downstream with the $x$-directed flow because momentum is transferred to the blob from the background. However if we examine the trajectory of the centre of mass for the blobs, an asymmetry in the $y$ direction becomes apparent. Figure 9 reveals that blobs with $R_{b} \sim \rho_{i}$ exhibit a small but measurable downward deflection of up to a full gyroradius over 5.5 gyroperiods, an order of magnitude greater than the negligible deflection for larger blobs seen in the largely symmetric trajectories in Figure 3. This is a result of the downward deflection of the background flow by the FLR symmetry breaking mechanism, which then causes momentum transfer in the $\mathbf{u} \times \mathbf{B}$ direction from the flow to the blob, pulling the blob plasma down. Local momentum transfer is mediated by the motional electric field associated with the locally dominant ion population. 

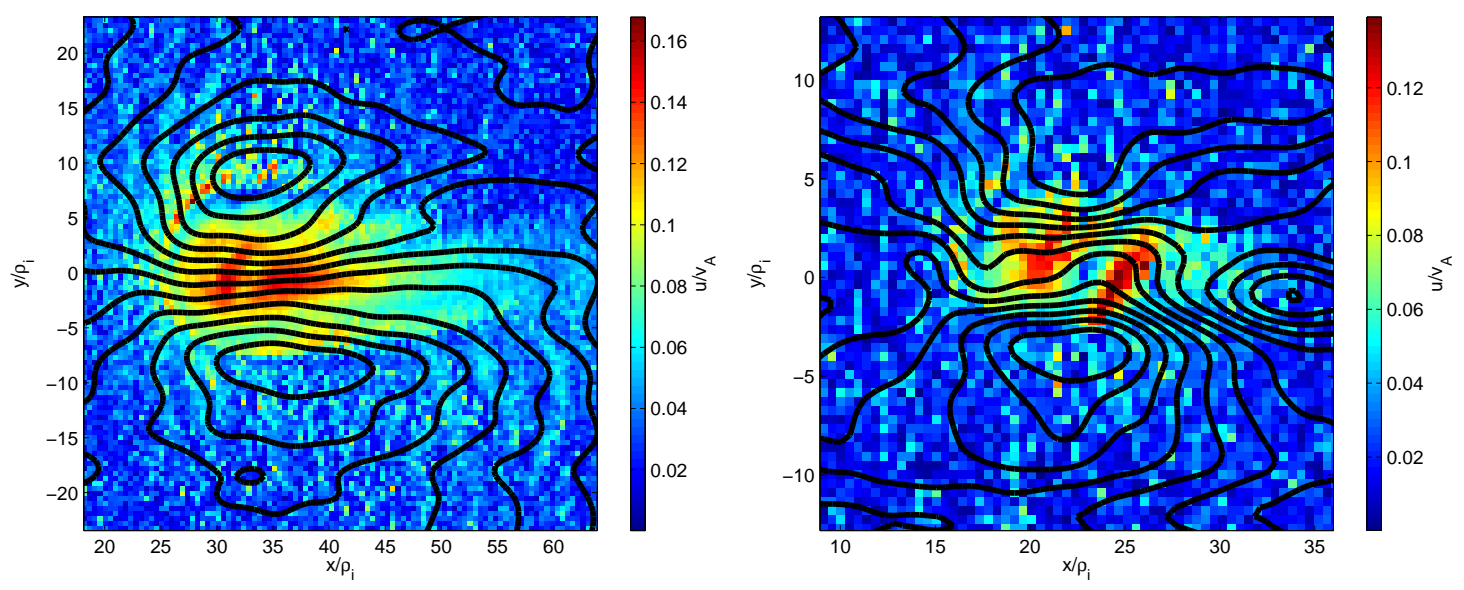

Figure 10. Approximate streamlines for blobs of radius $4 \rho_{i}$ (left) and $\rho_{i}$ (right) calculated under the assumption of incompressible flow, $\nabla \cdot \mathbf{u}=0$, after approximately 6 and 3 gyroperiods respectively. Streamlines are plotted in the rest frame of the flow, with the magnitude of the velocity also given in colour. The twin-celled convection pattern internal to the blob is asymmetric, with a larger cell on the lower edge than the upper edge.

We also see an asymmetry develop in the twin-celled convection pattern for the blobs with $R_{b} \sim \rho_{i}$. Due to the FLR symmetry breaking mechanism, the twin-vortex solution under a kinetic ion treatment deviates from the symmetric solution that would emerge from a purely fluid description. The difference in flow speeds between the upper and lower sides of the blob, which arises from the FLR symmetry breaking, causes the vorticity cells to grow asymmetrically, with a larger, faster flowing cell on the lower edge compared to a smaller, more slowly rotating cell on the upper edge. These asymmetric vorticity cells are shown in Figure 10, and are more pronounced for blobs of smaller radius. The difference in flow speeds at the leading edge of these vorticity cells contributes to the asymmetry which develops in the tails of the blobs for which $R_{b} \sim \rho_{i}$. Such blobs appear to have two tails, with a shorter tail forming on the upper edge, and a longer tail forming on the lower edge. For the smallest blob at $R_{b}=\rho_{i}$, no upper tail forms. This kind of clear asymmetry is not unique to these intermediate $R_{b} \sim \rho_{i}$ blobs, and has also been seen for obstacles with $R \ll \rho_{i}$. For example, the effect is visible in observations of the comet-like AMPTE solar wind releases [25]. 


\subsection{Kelvin-Helmholtz Instability}

At the boundaries between the blob and background plasmas, the sheared flow develops a Kelvin-Helmholtz $(\mathrm{K}-\mathrm{H})$ instability with structures on the ion gyroscale. The consequences of the instability are visible on the lower edges of the tails of the larger blobs in Figure 2, and more clearly for a large blob in Figure 11.

The magnetised fluid K-H instability arises when [35]

$$
\left(\mathbf{k} \cdot \mathbf{v}_{\mathbf{0}}\right)^{2}>\frac{n_{1}+n_{2}}{n_{1} n_{2}}\left[n_{1}\left(\mathbf{k} \cdot \mathbf{v}_{A 1}\right)^{2}+n_{2}\left(\mathbf{k} \cdot \mathbf{v}_{A 2}\right)^{2}\right]
$$

where $\mathbf{v}_{0}$ is the relative shear velocity, $n_{1,2}$ are the densities of the respective layers, and $v_{A 1,2}$ are the Alfvén speeds. The condition (10) is more easily satisfied for faster shear velocities, and is unconditionally satisfied when the magnetic fields are perpendicular to the shear, which is the case for these blob simulations. This instability enables momentum transfer between the two sheared fluids, contributing to the internal convection pattern described above.

An analytical expression for the growth rate of the Kevin-Helmholtz instability can be obtained for an MHD frame, with an infinitely thin boundary, incompressible media, and a simple geometry with velocities, magnetic field and tangential wave vector $k_{T}$ aligned in the same direction. In this case, the growth rate is simply proportional to $k_{T}$ [36]. However, when accounting for compressibility and a more complex geometry, numerical and parametric studies are required. With the additional assumption of a finite boundary width $a$, such studies reveal the growth rate reaches a maximum value for $k a \approx 1$. Hence we expect the fastest growing mode to have a wavelength on the order of the radius of the blob.

An analytic expression can be derived for the growth rate of the $\mathrm{K}-\mathrm{H}$ instability for the case of an incompressible plasma with a finite Larmor radius and a finite thickness of the boundary layer [37]. The physics of finite ion Larmor radius enters through the magnetic viscosity $\nu=\rho_{\mathrm{i}}^{2} \Omega_{\mathrm{c}, \mathrm{i}} / 4$. We define the non-dimensional parameters $\omega^{*}=\omega L_{0} / v_{A 2}, k^{*}=k L_{0}, \alpha=\rho_{01} / \rho_{02}, M=u_{02} / v_{A 2}, s=v_{A 1} / v_{A 2}$, and $\nu_{i}^{*}=\nu_{i} / v_{A i} L_{0}$; here $\rho_{0 i}$ is the density in layer $i$, and $L_{0}$ is the characteristic length. The growth rate $\Gamma^{*}$ is the real part of $\omega^{*}$, as derived in [37], given by:

$$
\begin{aligned}
\Gamma^{*}= & \frac{k^{*}}{\alpha+1}\left[(\alpha+1) M\left(M+(-1)^{j} 2 \nu_{2}^{*} k^{*}\right)\right. \\
& \left.-\left(M-(-1)^{j}\left(\alpha \nu_{1}^{*} s-\nu_{2}^{*}\right) k^{*}\right)^{2}\right]^{1 / 2} .
\end{aligned}
$$




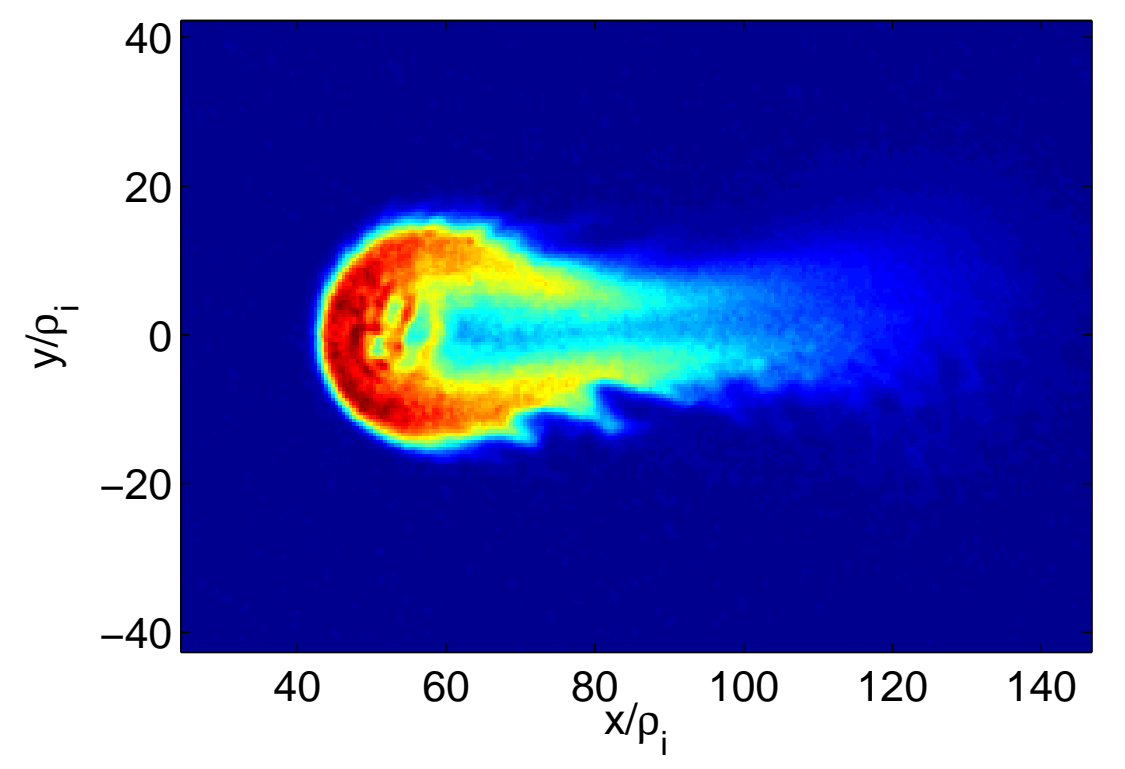

Figure 11. Number density plot for a blob of radius $R_{b}=10 \rho_{i}$ after 11 gyroperiods, demonstrating the asymmetric growth of a Kelvin-Helmholtz instability on the upper and lower edges of the blob, and in the tail. Note that the instability has grown much more quickly on the lower edge, and with associated structures whose size is much larger than those on the upper edge.

Plots of the theoretical growth rates $\Gamma^{*}$, calculated using (11), for the parameters used in our simulations of a blob of radius $10 \rho_{i}$ are given in Figure 12. The graph shows this analysis predicts a large disparity between the $\mathrm{K}-\mathrm{H}$ growth rates and the characteristics of the fastest growing modes that arise on the upper and lower edges of the blobs. On the lower edge, (11) predicts a faster growth rate of the instability over all values of $k$, higher than that expected under MHD assumptions, for which $\Gamma^{*}$ is proportional to $k^{*}$. For example, for a blob of radius $10 \rho_{i}$ this model predicts a sharp cut off at $k^{*}=6$, corresponding to a fastest growing mode with $\lambda=L_{0}$ which has a growth rate roughly 50 times greater than the fastest growing mode for the upper edge. The scale size of the fastest growing mode for the upper edge is, from this model, expected to be approximately an order of magnitude less than that for the lower edge. This large disparity in the scale sizes of the structures formed by the $\mathrm{K}-\mathrm{H}$ instability can be observed for our simulations in Figure 11. 


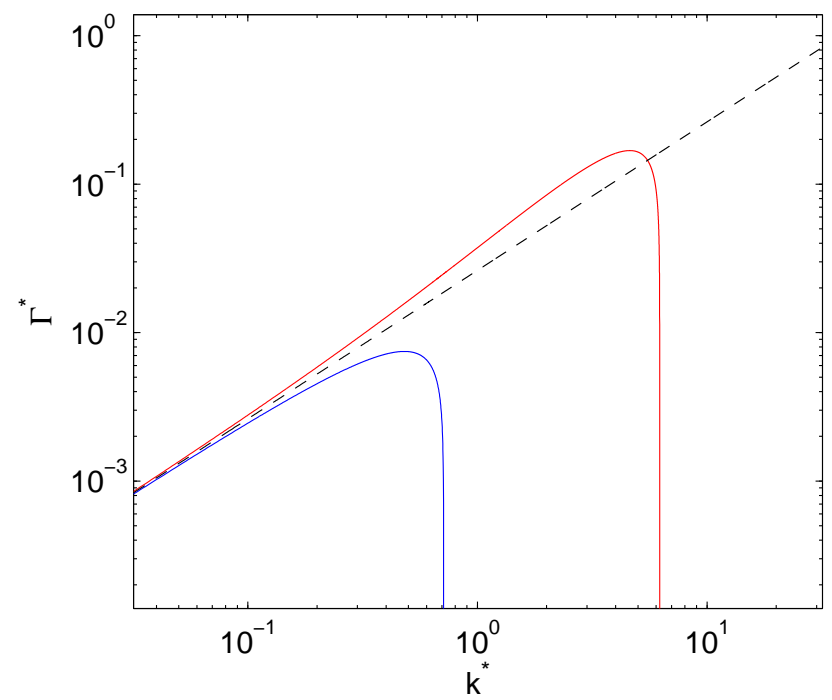

Figure 12. Growth rate $\Gamma^{*}$ of the $\mathrm{K}-\mathrm{H}$ instability for an incompressible plasma with the same flow speed on the upper and lower edges. $\Gamma^{*}$ is plotted against dimensionless wavenumber $k^{*}$ for the lower edge (red); upper edge (blue); and under MHD assumptions (dashed line). These theoretical growth rates have been calculated for a blob of radius $10 \rho_{i}$.

\section{Conclusions}

The preceding results demonstrate how the hybrid model (fluid electrons and fully kinetic ions) embodied in the code captures key elements of the plasma dynamics and the evolution of the fields in ion gyro-scale blobs. Many of the phenomena described above are common to a purely fluid approach to blob simulation, including the advection of blobs with the background flow, the development of a twin-celled vortex structure internal to the blob, and the growth of a Kelvin-Helmholtz instability in the tail formed from blob ejecta. However the substantial asymmetry which we find develops in these evolving features underlines the need to study small scale blob propagation using a hybrid method. The emergence of structures and gradients on ion gyro lengthscales, both internal to the blobs and at the boundary between the blob and external flowing plasma, further demonstrates the value of such a model. The resolution of ion gyro-scale physics deepens understanding of the mechanisms for momentum transport into and within the evolving blobs, in terms of both the 
motional electric fields and the non-linear phase of fluid-type instabilities such as Kelvin-Helmholtz.

With regard to the evolution of individual blobs, we conclude that symmetry breaking arising from finite ion Larmor radius effects plays a significant role in the evolution of blobs, in the case where inhomogeneities and their associated gradients cause significant variation in bulk properties over the lengthscale of a single ion gyration. The consequences of this asymmetry include: a poloidal deflection of the blob plasma in the $\mathbf{u} \times \mathbf{B}$ direction; a difference in size between the twin vortices generated internal to the blob; and a difference in the growth rate of the KelvinHelmholtz instability between the upper and lower edges of the blob, caused by both a growth of momentum in the background in the $\mathbf{u} \times \mathbf{B}$ direction, and by FLR effects at the shear boundary.

Some of these phenomena are known to arise for $R \ll \rho_{i}$ obstacles studied in a space science context. However the generation of the internal vortex cells, and their subsequent asymmetry and effect on the blob evolution, is unique to the intermediate scales chosen for this paper; comet-like objects for which $R \ll \rho_{i}$ cannot form such clear structures.

From the simulations presented, it is clear that the strength of the asymmetry is strongly dependent on the radius of the blob with respect to the ion gyroradius, and hence on the gradient lengthscales of the inhomogeneities associated with the blob. The form of the asymmetry of the evolution of the blob in the flow is also clearly dependent on the size of the blob relative to the ion gyroradius. Blobs with $R_{b} \gg \rho_{i}$ display an asymmetry that is most strongly conditioned by FLR effects on the K-H instability in the tail, while blobs with $R_{b} \sim \rho_{i}$ are affected more strongly by asymmetric deflection of the flow at the upstream edge.

Since ion gyro-scale blobs in the edge plasma are not easily resolved by current tokamak diagnostics, they constitute a population of structures which is likely to exist, but whose number and distribution are at present unknown. Further investigation of the effects of small blobs, in particular on energy dissipation within, and transport across, the edge plasma is hence desirable. Hybrid simulations will also assist the comparison between theory and observation as structures on this scale become resolved by future diagnostic advances. 


\section{Acknowledgments}

This work was part-funded by the EPSRC and the RCUK Energy Programme under grant EP/I501045 and the European Communities under the contract of Association between EURATOM and CCFE. The views and opinions expressed herein do not necessarily reflect those of the European Commission. We also thank the EPOCH development team for their work on the PIC code adapted for this research.

\section{References}

[1] Boedo J A et al 2003 Physics of Plasmas 10 1670-77

[2] Grulke O, Terry J L, Labombard B and Zweben S J 2006 Physics of Plasmas 13012306

[3] Myra J R, D'Ippolito D A, Stotler D P, Zweben S J, Leblanc B P, Menard J E, Maqueda R J and Boedo J 2006 Physics of Plasmas 13092509

[4] Nold B, Conway G D, Happel T, Müller H W, Rohde V, Stroth U and the ASDEX Updgrate Team 2010 Plasma Phys. Control. Fusion 52065005

[5] Kirk A et al 2006 Plasma Phys. Control. Fusion 48 B433

[6] Windisch T, Grulke O, Naulin V and Klinger T 2011 Plasma Phys. Control. Fusion 53085001

[7] Krasheninnikov S I 2001 Phys. Lett. A. 283 368-70

[8] D'Ippolito D A, Myra J R and Krasheninnikov S I 2002 Physics of Plasmas 9 222-33

[9] Aydemir A Y 2005 Physics of Plasmas 12062503

[10] Russel D A, D'Ippolito D A, Myra J R, Nevins W M and Xu X Q 2004 Phys. Rev. Lett. 93 265001

[11] Garcia O E, Bian N H, Naulin V, Nielsen A H and Rasmussen J J 2005 Physics of Plasmas 12090701

[12] Yu G Q, Krasheninnikov S I and Guzdar P N 2006 Physics of Plasmas 13042508

[13] Higgins D, Hnat B, Kirk A, Tamain P, Ben Ayed N and the MAST Team 2012 Plasma Phys. Control. Fusion 54015002

[14] Krasheninnikov S I 2011 Plasma Phys. Control. Fusion 53074017

[15] Chodura R 1975 Nucl. Fusion 15 55-61

[16] Auer P L, Kilb R W and Crevier W F 197176 2927-39

[17] Leroy M M, Goodrich C C, Winske D, Wu C S and Papadopoulos K 1981 Geophys. Res. Lett. 8 1269-72

[18] Chapman S C, Lee R E and Dendy R O 2005 Space Sci. Rev. 121 5-19

[19] Yuan X, Cairns I H, Trichtchenko L, Rankin R and Danskin D W 2009 Geophys. Res. Lett. 365103

[20] Swift D W 1995 Geophys. Res. Lett. 22 311-14

[21] Motschmann U and Kürt E 2006 Space Sci. Rev 122 197-208

[22] Brecht S H and Ferrante J R 1991 J. Geophys. Res. 9611209

[23] Lipatov A S, Sauer K and Baumgärtel K 1997 Advances in Space Research 20279

[24] Simon S and Motschmann U 2009 Planet. Space Sci. 57 2001-15 
[25] Valenzuela A, Haerendel G, Foeppl H, Melzner F and Neuss H 1986 Nature 320 700-3

[26] Chapman S C and Dunlop M W 1986 J. Geophys. Res. 91 8051-55

[27] Hesse M and Winske D 1994 J. Geophys. Res. 991 11177-92

[28] Birn J et al 2005 Geophys. Res. Lett. 3206105

[29] Yin L, Winske D, Gary S P and Birn J 2001 J. Geophys. Res 106 10761-76

[30] Karimabadi H, Krauss-Varban D, Huba J D and Vu H X 2004 J. Geophys. Res. 1099205

[31] Park W, Belova E V, Fu X Z, Strauss H R, Sugiyama L E 1999 Physics of Plasmas 6 1796-803

[32] Winske D, Yin L, Omidi N Karimabadi H and Quest K 2003 Space Plasma Simulation (Berlin: Springer-Verlag) 615 p 136-65

[33] Hewett D W 1994 Computer Phys. Communications 84 243-77

[34] Hill M J M 1894 Royal Society of London Philosophical Transactions Series A 185 213-45

[35] Treumann R A and Baumjohann W 1997 Advanced Space Plasma Physics (London: Imperial College Press) p 43-51

[36] Belmont G and Chanteur G 1989 Phys. Scr. 40 124-8

[37] Nagano H 1979 Planet. Space Sci. 27 881-4 